\title{
PARAMETRIZATIONS OF TEICHMÜLLER SPACES BY TRACE FUNCTIONS AND ACTION OF MAPPING CLASS GROUPS
}

\author{
GOU NAKAMURA AND TOSHIHIRO NAKANISHI \\ Dedicated to the memory of Professor Mika Seppälä
}

\begin{abstract}
We give a set of trace functions which give a global parametrization of the Teichmüller space $\mathcal{T}(g, n)\left(L_{1}, \ldots, L_{n}\right)$ of hyperbolic surfaces of genus $g$ with $n$ geodesic boundary components of lengths $L_{1}, \ldots, L_{n}$ such that the action of the mapping class group on the Teichmüller space can be represented by rational transformations in the parameters.
\end{abstract}

\section{INTRODUCTION}

Let $\bar{S}=\bar{S}_{g, n}$ be a surface of type $(g, n)$, a smooth compact and oriented surface of genus $g$ with $n$ boundary curves $C_{1}, \ldots, C_{n}$, and $S$ the interior of $\bar{S}$. Throughout this paper we assume that $2 g-2+n>0$. If $n \geq 1$, let $L=\left(L_{1}, \ldots, L_{n}\right)$ be a tuple of non-negative numbers. The Teichmüller space $\mathcal{T}(g, n)\left(L_{1}, \ldots, L_{n}\right)$ is the space of equivalence classes of marked complete hyperbolic metrics of curvature -1 on $S$ such that $C_{j}$ is homotopic to a closed geodesic curve of length $L_{j}$ in $S$ for $j=1, \ldots, n$ (If $L_{j}=0$, then $C_{j}$ corresponds to a puncture). We mean by $\mathcal{T}(g, 0)(L)$ the Teichmüller space of closed hyperbolic surfaces of genus $g$ whatever $L$ is. It is known that $\mathcal{T}(g, n)(L)$ is a real analytic manifold homeomorphic to $\mathbb{R}^{6 g-6+2 n}$ (see, for example, [22, Section 34]).

The free homotopy class of a homotopically non-trivial closed curve $c$ in $S$ defines a real analytic function $\ell_{c}$ on $\mathcal{T}(g, n)(L)$, called the geodesic length function of $c$, which assigns to each point in $\mathcal{T}(g, n)(L)$ the length of the geodesic curve freely homotopic to $c$ on a marked hyperbolic surface representing the point. It is known that there are finitely many closed curves $c_{1}, \ldots, c_{N}$ on $S$ such that each point of $\mathcal{T}(g, n)(L)$ is recovered by the values of their geodesic length functions (see Section 3.2.) In other words,

$$
\left(\ell_{c_{1}}, \ldots, \ell_{c_{N}}\right): \mathcal{T}(g, n)(L) \rightarrow \mathbb{R}_{>0}^{N} \quad\left(\mathbb{R}_{>0}=\{x \in \mathbb{R}: x>0\}\right)
$$

is a (real analytic) embedding (see, for example, [4, [1], [16, [17] and [18]). Then the problem of finding the minimal number $N(g, n)$ of geodesic length functions needed to parametrize $\mathcal{T}(g, n)(L)$ globally naturally arises. This problem was solved (Schmutz [15], Okumura [12]) and

$$
N(g, n)=6 g-5+2 n=\operatorname{dim} \mathcal{T}(g, n)(L)+1 .
$$

The fact that $N(g, n)>\operatorname{dim} \mathcal{T}(g, n)(L)$ follows from convexity of geodesic length functions (Wolpert [21]).

Received by the editors July 2, 2015 and, in revised form, January 11, 2016.

2010 Mathematics Subject Classification. Primary 32G15; Secondary 30F35.

The first author was partially supported by the JSPS KAKENHI Grant No. 25400147.

The second author was partially supported by the JSPS KAKENHI Grant No. 22540191. 
Let $G=G(g, n)$ be the fundamental group of $S$. Then $G$ has a generator system

$$
\mathscr{S}=\left(A_{1}, B_{1}, \ldots, A_{g}, B_{g}, C_{1}, \ldots, C_{n}\right)
$$

satisfying

$$
\left(\prod_{j=1}^{g} A_{j} B_{j} A_{j}^{-1} B_{j}^{-1}\right) C_{1} \cdots C_{n}=1,
$$

where $\left(A_{1}, B_{1}\right), \ldots,\left(A_{g}, B_{g}\right)$ correspond to handles and $C_{1}, \ldots, C_{n}$ to the boundary curves of $S$. By the uniformization theorem and the lifting theorem ([5], 2], 19]), we may think of $\mathcal{T}(g, n)\left(L_{1}, \ldots, L_{n}\right)$ as the space of conjugacy classes of faithful and discrete (or Fuchsian) representations $\rho$ of $G$ into $S L(2, \mathbb{R})$ by fixing the signs of $\rho(X)$ for $X \in \mathscr{S}$ so that $\rho\left(\left(\prod_{j=1}^{g} A_{j} B_{j} A_{j}^{-1} B_{j}^{-1}\right) C_{1} \cdots C_{n}\right)=1$ (the identity matrix).

Each $g \in G$ defines a trace function, $\chi_{g}([\rho])=\operatorname{tr} \rho(g)$ on $\mathcal{T}(g, n)(L)$. Due to (2.1) below we can replace geodesic length functions by trace functions. We rephrase the solution of the problem mentioned above.

Theorem 1.1 ([12, 15]). There exist $N=N(g, n)=6 g+2 n-5$ elements $g_{1}, \ldots$, $g_{N}$ in $G$ such that

$$
\left(\chi_{g_{1}}, \ldots, \chi_{g_{N}}\right): \mathcal{T}(g, n)\left(L_{1}, \ldots, L_{n}\right) \rightarrow \mathbb{R}^{N}
$$

is a real analytic embedding.

By a suitable choice of $g_{1}, \ldots, g_{N}$, the image of $\mathcal{T}(g, n)(L)$ under the embedding $\left(\chi_{g_{1}}, \ldots, \chi_{g_{N}}\right)$ is contained in the zero locus of an RC function (compass and ruler constructible function) (Feng Luo [6]).

The mapping class group $\mathcal{M C}(g, n)$ of $S$ acts on $\mathcal{T}(g, n)(L)$. So it is possible to describe each mapping class of $\mathcal{M C}(g, n)$ by using the parameters in $\mathbb{R}^{N}$. Our main theorem is

Theorem 1.2. There exist $N=N(g, n)$ elements $g_{1}, \ldots, g_{N}$ in $G$ such that

$$
\chi=\left(\chi_{g_{1}}, \ldots, \chi_{g_{N}}\right): \mathcal{T}(g, n)\left(L_{1}, \ldots, L_{n}\right) \rightarrow \mathbb{R}^{N}
$$

is a real analytic embedding and that each $\varphi \in \mathcal{M C}(g, n)$ acts on $\chi(\mathcal{T}(g, n)(L))$ as a rational transformation in the parameters of $\mathbb{R}^{N}$ and $c_{j}=2 \cosh \left(L_{j} / 2\right), j=$ $1, \ldots, n$.

In this note, a rational function in $x=\left(x_{1}, \ldots, x_{m}\right)$ means a function written in the form $P(x) / Q(x)$, where $P(x)$ and $Q(x)$ are integer polynomials. We denote the set of rational functions in $\left(x_{1}, \ldots, x_{m}\right)$ by $\mathscr{R}\left(x_{1}, \ldots, x_{m}\right)$. The theorem means that, if $x=\left(x_{1}, \ldots, x_{N}\right) \in \chi(\mathcal{T}(g, n)(L))$, then $\varphi(x)=\left(\varphi_{1}(x), \ldots, \varphi_{N}(x)\right) \in$ $\mathscr{R}\left(x_{1}, \ldots, x_{N}, c_{1}, \ldots, c_{n}\right)^{N}$.

For $(g, n)=(0,4)$ and $(1,1)$ this is a classical theorem. For example, $\mathcal{T}(1,1)(0)$ is identified with $\left\{(x, y, z): x>2, y>2, z>0, x^{2}+y^{2}+z^{2}=x y z\right\}$ and $\mathcal{M C}(1,1)$ with the group of Markov transformations, which are generated by

$$
\begin{aligned}
& \varphi_{*}(x, y, z)=\left(x, z, y^{-1}\left(x^{2}+z^{2}\right)\right)=(x, z, x z-y), \\
& \psi_{*}(x, y, z)=\left(z, y, x^{-1}\left(y^{2}+z^{2}\right)\right)=(z, y, y z-x) .
\end{aligned}
$$

(See [13, Example in p. 335]. It is known that the orbit of $(3,3,3)$ under $\mathcal{M C}(1,1)$ provides all positive integer solutions of $x^{2}+y^{2}+z^{2}=x y z$.) Our theorem may be viewed as a generalization of this result. 


\section{Preliminaries}

2.1. The Teichmüller space. Our basic references for notation and terminology about Fuchsian groups and Teichmüller spaces are [1, 20] and [22]. The group $P S L(2, \mathbb{R})$ acts on the Riemann sphere $\overline{\mathbb{C}}$ by Möbius transformations. If its action is restricted to $\mathbb{H}=\{z=x+i y: y>0\}$, a model of a hyperbolic plane, then it is the group of orientation-preserving isometries. A Fuchsian group $\Gamma$ is a discrete subgroup of $P S L(2, \mathbb{R})$. If $\Gamma$ is torsion free, then the space $\mathbb{H} / \Gamma$ is a hyperbolic surface. A hyperbolic element $A$ of $\Gamma$ has two fixed points $p_{A}$ and $q_{A}$ on the boundary $\partial \mathbb{H}$ in the Riemann sphere. The axis $L_{A}$ of $A$ is the hyperbolic line between $p_{A}$ and $q_{A}$. The cyclic group $\langle A\rangle$ generated by $A$ acts on $L_{A}$, and $L_{A} /\langle A\rangle$ is a closed geodesic curve $c_{A}$ on $\mathbb{H} / \Gamma$. The trace of $A$ and the length $\ell(A)=\ell_{c_{A}}$ of $c_{A}$ (counting multiplicity) is related by

$$
|\operatorname{tr} A|=2 \cosh \frac{\ell(A)}{2}
$$

Let $G=G(g, n)$ be the fundamental group of $S_{g, n}$. We fix a tuple $\left(c_{1}, \ldots, c_{n}\right)$ of positive numbers $\geq 2$. We consider faithful representations (injective homomorphisms) $\rho$ from $G$ into $S L(2, \mathbb{R})$ satisfying

$(*)\left\{\begin{array}{l}\rho(G) \text { is a Fuchsian group and } \mathbb{H} / \rho(G) \text { a surface of type }(g, n) \\ c_{j}=-\operatorname{tr} \rho\left(C_{j}\right), j=1, \ldots, n, \text { and if } c_{j}=2, \text { then } \rho\left(C_{j}\right) \text { is parabolic. } \\ \operatorname{tr} \rho\left(A_{j}\right)>0 \text { and } \operatorname{tr} \rho\left(B_{j}\right)>0 \text { for } j=1, \ldots, g\end{array}\right.$

We remark that for all faithful Fuchsian representations $\tilde{\rho}$ of $G$ into $P S L(2, \mathbb{R})$ with $c_{j}=\left|\operatorname{tr} \rho\left(C_{j}\right)\right|$, there exists a lift $\rho: G \rightarrow S L(2, \mathbb{R})$ of $\tilde{\rho}$ satisfying the above conditions (see the proofs of Theorems 3.5 and 3.6 in [19]). Two representations $\rho_{1}$ and $\rho_{2}$ satisfying $(*)$ are equivalent if there exists a $P \in S L(2, \mathbb{R})$ such that $\rho_{2}(g)=P^{-1} \rho_{1}(g) P$ for all $g \in G$.

Definition 2.1. The Teichmüller space $\mathscr{T}(g, n)\left(c_{1}, \ldots, c_{n}\right)$ is the space of equivalence classes of faithful Fuchsian representations which satisfy $(*)$.

By the uniformization theorem, this space is identified with $\mathcal{T}(g, n)\left(L_{1}, \ldots, L_{n}\right)$ in the introduction, if $c_{j}=2 \cosh \left(L_{j} / 2\right)$. For $[\rho] \in \mathscr{T}(g, n)\left(c_{1}, \ldots, c_{n}\right), \rho$ is determined by the images of generators in (1.2). So we can identify $[\rho]$ with the simultaneous conjugacy class of the tuple $\left(\rho\left(A_{1}\right), \rho\left(B_{1}\right), \ldots, \rho\left(C_{n}\right)\right)$.

2.2. Trace identities for $S L(2, \mathbb{R})$. We list basic trace identities for matrices in $S L(2, \mathbb{R})$ (see, for example, [7, $\S 3.4]$ ):

(I1) $\operatorname{tr} A_{1} A_{2} \cdots A_{n}=\operatorname{tr} A_{i_{1}} A_{i_{2}} \cdots A_{i_{n}}$, where $\left(i_{1}, \ldots, i_{n}\right)$ is a cyclic permutation of $(1, \ldots, n)$.

(I2) $\operatorname{tr} A=\operatorname{tr} A^{-1}$, hence, if $A B=1$ (the identity matrix), then $\operatorname{tr} A=\operatorname{tr} B$.

(I3) $\operatorname{tr} A B+\operatorname{tr} A B^{-1}=\operatorname{tr} A \operatorname{tr} B$, hence $\operatorname{tr} A B^{-1} C=\operatorname{tr} B \operatorname{tr} A C-\operatorname{tr} A B C$.

(I4) $\operatorname{tr} A B C=\operatorname{tr} A \operatorname{tr} B C+\operatorname{tr} B \operatorname{tr} C A+\operatorname{tr} C \operatorname{tr} A B-\operatorname{tr} A \operatorname{tr} B \operatorname{tr} C-\operatorname{tr} A C B$.

(I5) For the commutator $[A, B]=A B A^{-1} B^{-1}$,

$$
\operatorname{tr}[A, B]=(\operatorname{tr} A)^{2}+(\operatorname{tr} B)^{2}+(\operatorname{tr} A B)^{2}-\operatorname{tr} A \operatorname{tr} B \operatorname{tr} A B-2 .
$$


(I6) $2 \operatorname{tr} A B C D=(\operatorname{tr} A)(\operatorname{tr} B C D)+(\operatorname{tr} B)(\operatorname{tr} A C D)+(\operatorname{tr} C)(\operatorname{tr} A B D)$

$$
\begin{aligned}
& +(\operatorname{tr} D)(\operatorname{tr} A B C)+(\operatorname{tr} A B)(\operatorname{tr} C D)-(\operatorname{tr} A C)(\operatorname{tr} B D) \\
& +(\operatorname{tr} A D)(\operatorname{tr} B C)-(\operatorname{tr} A)(\operatorname{tr} B)(\operatorname{tr} C D)-(\operatorname{tr} B)(\operatorname{tr} C)(\operatorname{tr} A D) \\
& -(\operatorname{tr} C)(\operatorname{tr} D)(\operatorname{tr} A B)-(\operatorname{tr} A)(\operatorname{tr} D)(\operatorname{tr} B C) \\
& +(\operatorname{tr} A)(\operatorname{tr} B)(\operatorname{tr} C)(\operatorname{tr} D) .
\end{aligned}
$$

(I7) $\operatorname{tr} A^{-1} B A C=\operatorname{tr} A \operatorname{tr} B A C+\operatorname{tr} B \operatorname{tr} C-\operatorname{tr} A B \operatorname{tr} A C-\operatorname{tr} B C$.

The identity (I7) is not listed in [7, §3.4], but it is a consequence of (I1)-(I4):

$$
\begin{aligned}
\operatorname{tr} A^{-1}(B A) C= & \operatorname{tr} A^{-1} \operatorname{tr} B A C+\operatorname{tr} B A \operatorname{tr} A^{-1} C+\operatorname{tr} C \operatorname{tr} A^{-1} B A \\
& -\operatorname{tr} A^{-1} \operatorname{tr} B A \operatorname{tr} C-\operatorname{tr}(B A) A^{-1} C \\
= & \operatorname{tr} A \operatorname{tr} B A C+\operatorname{tr} A B(\operatorname{tr} A \operatorname{tr} C-\operatorname{tr} A C)+\operatorname{tr} B \operatorname{tr} C \\
& -\operatorname{tr} A \operatorname{tr} A B \operatorname{tr} C-\operatorname{tr} B C .
\end{aligned}
$$

Definition 2.2. A tuple $\mathscr{S}=\left(A_{1}, B_{1}, \ldots, A_{g}, B_{g}, C_{1}, \ldots, C_{n}\right)$ of matrices in $S L(2, \mathbb{R})$ is said to be of type $(g, n)$ if $\mathscr{S}$ represents a point of $\mathscr{T}_{g, n}\left(c_{1}, \ldots, c_{n}\right)$ with $c_{j}=-\operatorname{tr} C_{j}, j=1, \ldots, n$.

Let $(A, B, C, D)$ be of type $(0,4)$. So it satisfies $A B C D=1$. Let $a=-\operatorname{tr} A$, $b=-\operatorname{tr} B, c=-\operatorname{tr} C, d=-\operatorname{tr} D, x=-\operatorname{tr} B C, y=-\operatorname{tr} C A$ and $z=-\operatorname{tr} A B$ and

$$
\Sigma_{04}=\{x, y, z, a, b, c, d\} \text {. }
$$

Then (see [6], 9])

$$
\begin{aligned}
& F_{04}(x, y, z, a, b, c, d) \\
& =x^{2}+y^{2}+z^{2}-x y z+(a d+b c) x+(b d+c a) y+(c d+a b) z \\
& \quad+a^{2}+b^{2}+c^{2}+d^{2}+a b c d-4 \\
& =0 .
\end{aligned}
$$

For an ordered tuple $\mathscr{S}=\left(A_{1}, \ldots, A_{n}\right)$ of matrices in $S L(2, \mathbb{R})$ we define

$$
T(\mathscr{S})=\left\{\begin{array}{l|l}
A_{i}, & 1 \leq i \leq n, \\
A_{j_{1}} A_{j_{2}}, & 1 \leq j_{1}<j_{2} \leq n, \\
A_{k_{1}} A_{k_{2}} A_{k_{3}} & 1 \leq k_{1}<k_{2}<k_{3} \leq n
\end{array}\right\} .
$$

If $G(\mathscr{S})$ denotes the group generated by $A_{1}, \ldots, A_{n}$, then

Lemma 2.3 (see [7, Lemma 3.5.3]). If $g \in G(\mathscr{S})$, then its trace $\operatorname{tr} g$ is a rational polynomial in $\{\operatorname{tr} g: g \in T(\mathscr{S})\}$.

Remark. The results in this subsection hold for $S L(2, \mathbb{C})$.

\section{Parametrization of the Teichmüller spaces}

3.1. Teichmüller spaces of types $(1,1)$ and $(0,4)$. For the results of this subsection we refer to [14, [10, [3] and [22, §33]. We often use

Lemma 3.1 (22, Lemmas 33.4 and 33.17]). If $(A, B, C), A B C=1$, is of type $(0,3)$, then $(\operatorname{tr} A)(\operatorname{tr} B)(\operatorname{tr} A B)<0$. If $(A, B, C),[A, B] C=1$, is of type $(1,1)$, then $(\operatorname{tr} A)(\operatorname{tr} B)(\operatorname{tr} A B)>0$ and $\operatorname{tr}[A, B]=x^{2}+y^{2}+z^{2}-x y z-2$ is negative.

$\mathcal{T}(0,3)(a, b, c)$ is a one point set ([22, Proposition 33.6]). Next, let $(A, B, C)$, $[A, B] C=1$, be a tuple of type $(1,1)$ representing a point of $\mathcal{T}(1,1)(c)$. Since $A$ and $B$ have positive trace, $\operatorname{tr} A B>0$ by Lemma 3.1. Let $x=\operatorname{tr} A, y=\operatorname{tr} B$ and $z=\operatorname{tr} A B$. Then $(x, y, z)$ embeds $\mathcal{T}(1,1)(c)$ into $\mathbb{R}^{3}$ and its image is

$$
\left\{(x, y, z): x>2, y>2, z>2, x y z-x^{2}-y^{2}-z^{2}+2=c\right\}
$$


[22, §33.D]. Next, let $(A, B, C, D)$ be a tuple of type $(0,4)$ representing a point of $\mathcal{T}(0,4)(a, b, c, d)$. By condition $(*), a=-\operatorname{tr} A, b=-\operatorname{tr} B, c=-\operatorname{tr} C$ and $d=-\operatorname{tr} D$ are positive. We define $x=-\operatorname{tr} B C, y=-\operatorname{tr} C A$, and $z=-\operatorname{tr} A B$. These values are all positive. For example, we know $\operatorname{tr} B C<0$ by applying Lemma 3.1 to the tuple $(B, C, D A)$ of type $(0,3)$ with $\operatorname{tr} B<0, \operatorname{tr} C<0$. We see that $(x, y, z)$ embeds $\mathcal{T}(0,4)(a, b, c, d)$ into $\mathbb{R}^{3}$, and its image is

$$
\left\{(x, y, z): x>2, y>2, z>2, F_{04}(x, y, z, a, b, c, d)=0\right\},
$$

where $F_{04}$ is given in (2.3) $\left.(10]\right)$.

3.2. Geometric transformations on the tuples of type $(g, n)$. We need the following lemma.

Lemma 3.2. For tuples $\left(F_{1}, \ldots, F_{2 g+n}\right)$ of type $(g, n)$ with $n \geq 2$ satisfying

$$
\left(\prod_{k=1}^{g}\left[F_{2 k-1}, F_{2 k}\right]\right) F_{2 g+1} \cdots F_{2 g+n}=1
$$

and for $k=2 g+1, \ldots, 2 g+n$,

$$
\begin{aligned}
& \sigma_{k}\left(F_{1}, \ldots, F_{k}, F_{k+1}, \ldots, F_{2 g+n}\right)=\left(F_{1}, \ldots, F_{k} F_{k+1} F_{k}^{-1}, F_{k}, \ldots, F_{2 g+n}\right) \\
& \sigma_{k}^{-1}\left(F_{1}, \ldots, F_{k}, F_{k+1}, \ldots, F_{2 g+n}\right)=\left(F_{1}, \ldots, F_{k+1}, F_{k+1}^{-1} F_{k} F_{k+1}, \ldots, F_{2 g+n}\right)
\end{aligned}
$$

are also of type $(g, n)$.

In fact, $\sigma_{k}$ arises from a Dehn twist of the loop on the surface $S_{g, n}$ bounding the $(k-2 g)$-th and $(k+1-2 g)$-th boundary curves.

3.3. Parametrization of Teichmüller spaces. It is known that there are trace functions $\chi_{g_{1}}, \ldots, \chi_{g_{N}}$ which embed the Teichmüller space $\mathcal{T}(g, n)\left(c_{1}, \ldots, c_{n}\right)$ into $\mathbb{R}^{N}$. For example, choose $g_{11}, \ldots, g_{m 1} \in G(g, n)$ representing homotopy classes of simple closed curves $\gamma_{1}, \ldots, \gamma_{m}, m=3 g-3+n$, which give a pants-decomposition of $S$. For each $j=1, \ldots, m$, choose a simple closed curve $\delta_{j}$ which meets $\gamma_{j}$ once if $\gamma_{j}$ is non-separating or twice if $\gamma_{j}$ is separating. Let $\eta_{j}$ be a curve obtained from $\delta_{j}$ by a Dehn twist along $\gamma_{j}$. Then, as the cases of types $(1,1)$ and $(0,4)$ indicate, the geodesic length functions on $\gamma_{j}, \eta_{j}$ and $\delta_{j}$ determine the length and twist parameters along $\gamma_{j}$ in the Fenchel-Nielsen coordinates. So, if $g_{j 2}$ and $g_{j 3}$ are the homotopy classes of $\delta_{j}$ and $\eta_{j}$, respectively, then the trace functions $\chi_{g_{j k}}$ $(j=1, \ldots, m, k=1,2,3)$ embed $\mathcal{T}(g, n)\left(L_{1}, \ldots, L_{n}\right)$ or $\mathscr{T}(g, n)\left(c_{1}, \ldots, c_{n}\right)$ with $c_{j}=2 \cosh \left(L_{j} / 2\right)$ into $\mathbb{R}^{3 m}$. Let $\mathscr{S}=\left(X_{1}, X_{2}, \ldots, X_{2 g+n}\right)$ represent a general point of $\mathscr{T}(g, n)\left(c_{1}, \ldots, c_{n}\right)$. Theorem 1.2 follows from Lemma 2.3 if we find $N=N(g, n)$ elements $g_{1}, \ldots, g_{N}$ in $G(\mathscr{S})$ such that the trace of each $g \in T(\mathscr{S})$ is a rational function in $\operatorname{tr} g_{1}, \ldots, \operatorname{tr} g_{N}$. The rest of paper is devoted to proving this.

\section{Proof of the main theorem: Cases of types $(0,5)$ and $(1,2)$}

4.1. Case of type $(0,5)$. Let $\mathscr{S}=(A, B, C, D, E)$ with $A B C D E=1$ be of type $(0,5)$ and $a=-\operatorname{tr} A, b=-\operatorname{tr} B, c=-\operatorname{tr} C, d=-\operatorname{tr} D$ and $e=-\operatorname{tr} E$. The group $G(\mathscr{S})$ is generated by $A, B, C$ and $D$. We define

$$
\Sigma_{05}=\{x, y, z, u, v, a, b, c, d, e\},
$$

where

$$
\begin{array}{ll}
x=-\operatorname{tr} B C, & y=-\operatorname{tr} C A, \quad z=-\operatorname{tr} A B, \\
u=-\operatorname{tr} B C D, & v=-\operatorname{tr} A D .
\end{array}
$$


Proposition 4.1. Traces of all elements in $G(\mathscr{S})$ are rational functions in $\Sigma_{05}$. Moreover, $\Sigma_{05}$ satisfies the polynomial identity $F_{05}\left(\Sigma_{05}\right)=0$ given in (4.5) below.

Proof. We shall show that traces of all elements in $T(\mathscr{S})$ in (2.4) are rational functions in $\Sigma$. The traces of elements in $T(\mathscr{S})$ not appearing in (4.1) are

$$
t=-\operatorname{tr} A B C, x_{1}=-\operatorname{tr} B D, x_{2}=-\operatorname{tr} C D, x_{3}=-\operatorname{tr} A B D, x_{4}=-\operatorname{tr} A C D .
$$

We have $\operatorname{tr} E=\operatorname{tr}(A B C D)^{-1}=\operatorname{tr} A B C D$, and by using (I7) and other trace identities we get

$$
\begin{array}{ll}
\operatorname{tr} A E=\operatorname{tr} B C D & =-u, \\
\operatorname{tr} B E=\operatorname{tr} B^{-1} E^{-1}=\operatorname{tr} B^{-1} A B(C D) & =b e+a x_{2}-z u+x_{4}, \\
\operatorname{tr} C E=\operatorname{tr} C^{-1} E^{-1}=\operatorname{tr} C^{-1}(A B) C D & =c e+z d-t x_{2}+x_{3}, \\
\operatorname{tr} D E=\operatorname{tr} A B C & =-t, \\
\operatorname{tr} A B E=\operatorname{tr} C D & =-x_{2}, \\
\operatorname{tr} A C E=\operatorname{tr} C^{-1} A^{-1} E^{-1}=\operatorname{tr} C^{-1} B C D & =c u+b d-x x_{2}+x_{1}, \\
\operatorname{tr} A D E=\operatorname{tr} B C & =-x, \\
\operatorname{tr} B C E=\operatorname{tr}(B C)^{-1} E^{-1}=\operatorname{tr}(B C)^{-1} A(B C) D & =x e+a d-t u+v, \\
\operatorname{tr} B D E=\operatorname{tr} D^{-1} B^{-1} E^{-1}=\operatorname{tr} B^{-1} A B C & =b t+a c-x z+y, \\
\operatorname{tr} C D E=\operatorname{tr} A B & =-z .
\end{array}
$$

We consider two tuples of type $(0,4),(A, B, C, D E)$ with

$$
\{-\operatorname{tr} B C,-\operatorname{tr} C A,-\operatorname{tr} A B,-\operatorname{tr} A,-\operatorname{tr} B,-\operatorname{tr} C,-\operatorname{tr} D E\}=\{x, y, z, a, b, c, t\}
$$

and $(A, B C, D, E)$ with

$$
\{-\operatorname{tr} B C D,-\operatorname{tr} D A,-\operatorname{tr} A B C,-\operatorname{tr} A,-\operatorname{tr} B C,-\operatorname{tr} D,-\operatorname{tr} E\}=\{u, v, t, a, x, d, e\} .
$$

From (2.3) we have two equations:

$$
F_{04}(x, y, z, a, b, c, t)=0, F_{04}(u, v, t, a, x, d, e)=0 .
$$

Solving $F_{04}(x, y, z, a, b, c, t)-F_{04}(u, v, t, a, x, d, e)=0$ in $t$, we see that

$$
t=\frac{P(a, b, c, d, e, x, y, z, u, v)}{Q(a, b, c, d, e, y, z, u, v)}
$$

where

$$
\begin{aligned}
P(a, b, c, d, e, x, y, z, u, v)= & a d e x+a e u+a d v+x y z+d u x+e v x-b c x-a c y \\
& -a b z+d^{2}+e^{2}+u^{2}+v^{2}-b^{2}-c^{2}-y^{2}-z^{2}
\end{aligned}
$$

and

$$
Q(a, b, c, d, e, y, z, u, v)=a b c-d e+u v+b y+c z .
$$

By substituting this with $t$ in $F_{04}(x, y, z, a, b, c, t)=0$, we obtain a polynomial identity:

$$
F_{05}\left(\Sigma_{05}\right)=F_{05}(x, y, z, u, v, a, b, c, d, e)=0 .
$$


The polynomial $F_{05}\left(\Sigma_{05}\right)$ is quadratic in $x$ and quartic and monic in $b, c, d, e, y$, $z, u$ and $v$. Next we consider $x_{1}, x_{2}, x_{3}$ and $x_{4}$. By (I6) and (4.2) we have

$$
\begin{aligned}
-2 b= & 2 \operatorname{tr} A C D E \\
= & \operatorname{tr} A \operatorname{tr} C D E+\operatorname{tr} C \operatorname{tr} A D E+\operatorname{tr} D \operatorname{tr} A C E+\operatorname{tr} E \operatorname{tr} A C D+\operatorname{tr} A C \operatorname{tr} D E \\
& -\operatorname{tr} A D \operatorname{tr} C E+\operatorname{tr} A E \operatorname{tr} C D-\operatorname{tr} A \operatorname{tr} C \operatorname{tr} D E-\operatorname{tr} C \operatorname{tr} D \operatorname{tr} A E-\operatorname{tr} D \operatorname{tr} E \operatorname{tr} A C \\
& -\operatorname{tr} E \operatorname{tr} A \operatorname{tr} C D+\operatorname{tr} A \operatorname{tr} C \operatorname{tr} D \operatorname{tr} E \\
= & a z+c x+d\left(x x_{2}-x_{1}-c u-b d\right)+e x_{4}+y t+v\left(c e+z d-t x_{2}+x_{3}\right) \\
& +u x_{2}+a c t+c d u+d e y+a e x_{2}+a c d e \\
= & -d x_{1}+(d x-v t+u+a e) x_{2}+v x_{3}+e x_{4} \\
& +a z+c x-b d^{2}+y t+v c e+v z d+a c t+d e y+a c d e .
\end{aligned}
$$

In a similar way we apply (I6) and (4.2) to $-c=\operatorname{tr} A B D E,-d=\operatorname{tr} A B C E$ and $-e=\operatorname{tr} A B C D$ to see that $x_{1}, x_{2}, x_{3}$ and $x_{4}$ satisfy

$$
\left(\begin{array}{cccc}
-d & u+d x-t v+a e & v & e \\
u+a e & d+a v & e & v \\
-b & c+b x+t z+a y+a b t & -z-a b & y \\
-y & z+a b & c & b
\end{array}\right)\left(\begin{array}{l}
x_{1} \\
x_{2} \\
x_{3} \\
x_{4}
\end{array}\right)
$$

$$
=\left(\begin{array}{c}
-2 b-a z-c x+b d^{2}-y t-c e v+d v z-a c t-d e y-a c d e \\
-2 c-a x z+a^{2} c+a y-b x-t z-b e v+z u v-b d u-d e z-a b d e \\
-2 d-a t u+a^{2} d+a v+b^{2} d-e t+d z^{2}-b e y+u y z-u x+a b d z \\
-2 e-a u-d t-v x-b c v-c d z-a d x-a b c d
\end{array}\right) .
$$

So far our calculations are in a formal manner. If $(A, B, C, D, E)$ represents a point of the Teichmüller space $\mathscr{T}(0,5)(a, b, c, d, e)$, the parameters in (4.1) and $t=-\operatorname{tr} A B C$ are greater than 2 , owing to the condition $(*)$ and that each of them corresponds to a boundary curve of a subsurface of type $(0,3)$ ([22, Lemma 33.4]). Since the quadratic equation

$$
F_{04}(u, v, t, a, x, d, e)=t^{2}-(u v-d e-a x) t+c_{0}=0,
$$

with

$$
c_{0}=u^{2}+v^{2}+(a e+x d) u+(x e+a d) v+a^{2}+x^{2}+d^{2}+e^{2}+a x d e-4
$$

positive, has a solution $t>2$, we must have $u v-d e>a x>0$. Therefore (4.3) holds, since its denominator (4.4) is positive over $\mathscr{T}(0,5)(a, b, c, d, e)$. As mentioned in Section 3.3. $(x, y, z, u, v, t)$ gives a global parameter system of $\mathscr{T}(0,5)(a, b, c, d, e)$ and now we can omit $t$. The traces $x_{1}, x_{2}, x_{3}$ and $x_{4}$ are real analytic functions on the Teichmüller space. Since $t$ in (4.3) is linear in $x$, the determinant of the matrix on the left-hand side of (4.6) is also a linear polynomial in $x$. By also using the quadratic equation (4.5) in $x$, we see that the determinant vanishes only in a lower dimensional subvariety in the four dimenisonal $(y, z, u, v)$-space. (The authors guess that the determinant never vanishes on the Teichmüller space.) So by solving (4.6) and by the continuity of the traces, we can express $x_{1}, x_{2}, x_{3}$ and $x_{4}$ as rational functions in $\{x, y, z, u, v, a, b, c, d, e\}$.

4.2. Case of type $(1,2)$. Let $\mathscr{S}=(A, B, C, D)$ be of type $(1,2)$. The matrices satisfy $[A, B] C D=1, c=-\operatorname{tr} C$ and $d=-\operatorname{tr} D$. 
4.2.1. We define $\Sigma_{12}^{(1)}=\{a, b, z, x, y, c, d\}$, where

$$
\begin{array}{lll}
a=\operatorname{tr} A, & b=\operatorname{tr} B, & d=-\operatorname{tr} D=-\operatorname{tr}[A, B] C, \\
z=\operatorname{tr} A B, & x=\operatorname{tr} B A^{-1} B^{-1} C, & y=\operatorname{tr} A C .
\end{array}
$$

Note that $a$ equals $\operatorname{tr} A$ but not $-\operatorname{tr} A$. This is the reason for the choice of $x$ and $y$.

Proposition 4.2. Let $A, B$ and $C$ be matrices in $S L(2, \mathbb{R})$ and suppose that $\mathscr{S}=$ $(A, B, C, D), D=C^{-1}[B, A]$ is of type $(1,2)$. Then

$$
x_{1}=\operatorname{tr} B C \quad \text { and } \quad x_{2}=\operatorname{tr} A B C
$$

are rational functions in $\Sigma_{12}^{(1)}$, and hence so are the traces of all elements in $G(\mathscr{S})$. The traces in $\Sigma_{12}^{(1)}$ satisfy the polynomial identity $F_{12}\left(\Sigma_{12}^{(1)}\right)=0$ given in (4.8) below.

Proof. Note that the traces of elements in $T(\mathscr{S})$ in (2.4) not appearing in (4.7) are $x_{1}$ and $x_{2}$. We have two linear equations in $x_{1}$ and $x_{2}$ :

$$
\begin{aligned}
x= & \operatorname{tr} B A^{-1} B^{-1} C=\operatorname{tr} B A^{-1} B^{-1} \operatorname{tr} C-\operatorname{tr} B^{-1} C B A(\because \text { (I3)) } \\
= & \operatorname{tr} A \operatorname{tr} C-(\operatorname{tr} B \operatorname{tr} C B A+\operatorname{tr} C \operatorname{tr} A-\operatorname{tr} C B \operatorname{tr} B A-\operatorname{tr} C A)(\because \text { (I7)) } \\
= & -b(\operatorname{tr} C \operatorname{tr} B A+\operatorname{tr} B \operatorname{tr} C A+\operatorname{tr} A \operatorname{tr} C B-\operatorname{tr} A \operatorname{tr} B \operatorname{tr} C-\operatorname{tr} A B C) \\
& +x_{1} z+y \quad(\because(\mathrm{I} 4)) \\
= & b c z-b^{2} y-a b x_{1}-a b^{2} c+b x_{2}+x_{1} z+y \\
= & -(a b-z) x_{1}+b x_{2}+b c z-b^{2} y-a b^{2} c+y, \\
-d= & \operatorname{tr} A B A^{-1} B^{-1} C=\operatorname{tr} A^{-1}\left(B^{-1} C\right) A B \\
= & \operatorname{tr} A \operatorname{tr} A C+\operatorname{tr} B^{-1} C \operatorname{tr} B-\operatorname{tr} B^{-1} C A \operatorname{tr} A B-\operatorname{tr} C \quad(\because(\mathrm{I} 7)) \\
= & a y+(\operatorname{tr} B \operatorname{tr} C-\operatorname{tr} B C) \operatorname{tr} B-(\operatorname{tr} B \operatorname{tr} A C-\operatorname{tr} A B C) \operatorname{tr} A B+c \\
& =-b x_{1}+z x_{2}-b y z+a y-b^{2} c+c .
\end{aligned}
$$

Since $\operatorname{tr}[A, B]<-2$ by Lemma 3.1

$$
\left|\begin{array}{cc}
a b-z & -b \\
b & -z
\end{array}\right|=\operatorname{tr}[A, B]-a^{2}+2<0 .
$$

Solving equations above, we may express $x_{1}$ and $x_{2}$ as rational functions in $\Sigma_{12}^{(1)}$.

The tuple $\left(-A,-B A^{-1} B^{-1}, C, D\right)$ is of type $(0,4)$. Its corresponding $\Sigma_{04}$ in (2.2) is $\left\{x, y, a b z-a^{2}-b^{2}-z^{2}+2, a, a, c, d\right\}$. So we obtain the identity

$$
F_{04}\left(x, y, a b z-a^{2}-b^{2}-z^{2}+2, a, a, c, d\right)=0 .
$$

The polynomial in the left-hand side is monic and quadratic in $c$ and $d$.

4.2.2. For our later use, we introduce the second system of traces [8, (3.2)],

$$
\Sigma_{12}^{(2)}=\{z, u, v, w, k, c, d\}
$$

where

$$
\begin{aligned}
& z=\operatorname{tr} A B, \quad u=\operatorname{tr} C A B A^{-1}, \quad v=\operatorname{tr} C A B^{2}, \quad w=\operatorname{tr} C A B \\
& k=-\operatorname{tr}[A, B]=-\operatorname{tr} C D,
\end{aligned}
$$

Proposition 4.3. Traces of all elements in $G(\mathscr{S})$ are rational functions in $\Sigma_{12}^{(2)}$. 
A proof of this proposition is given in [8, Section 3]. If $S^{2}=u v z-u^{2}-v^{2}-z^{2}$, then we have

$$
\begin{aligned}
& \operatorname{tr} A=\frac{w(u z-v)(c+d+z w)+u\left(2+c d+k+S^{2}+z^{2}-w^{2}\right)}{w\left(S^{2}+z^{2}\right)}, \\
& \operatorname{tr} B=\frac{u w(c+d+z w)+v\left(2+c d+k+S^{2}+z^{2}-w^{2}\right)}{w\left(S^{2}+z^{2}\right)}, \\
& \operatorname{tr} A C=b w-v, \\
& \operatorname{tr} B C=-b z w+z v+a w-b c-u .
\end{aligned}
$$

From [8, (3.10)], the parameters satisfy the polynomial identity

$$
F_{12}(u, v, w, z, k, c, d)=k^{2}+P k+Q=0,
$$

where

$$
\begin{aligned}
& P=2\left(w^{2}+\frac{c+d}{2} z w+S^{2}+z^{2}+c d+2\right)-w^{2}\left(S^{2}+4\right), \\
& Q=\left(w^{2}+\frac{c+d}{2} z w+S^{2}+z^{2}+c d+2\right)^{2}-2 w^{2}\left(S^{2}+4\right)-\left(\frac{c-d}{2}\right)^{2} w^{2}\left(z^{2}-4\right) .
\end{aligned}
$$

5. Cases of types $(0, n)$ AND $(1, n)$

5.1. type $(0, n)$. Let $\mathscr{S}=\left(A_{1}, A_{2}, \ldots, A_{n}\right)$ represent a point of the Teichmüller space $\mathscr{T}(0, n)\left(a_{1}, \ldots, a_{n}\right)$ with $n \geq 4$. The matrices $A_{1}, \ldots, A_{n}$ in $S L(2, \mathbb{R})$ satisfy $A_{1} A_{2} \cdots A_{n}=1$ and $a_{j}=-\operatorname{tr} A_{j}$ for $j=1, \ldots, n$. The group $G(\mathscr{S})$ is generated by $A_{1}, A_{2}, \ldots, A_{n-1}$. We define

$$
\Sigma_{0 n}=\left\{z, x_{3}, y_{3}, \ldots, x_{n-1}, y_{n-1}, a_{1}, \ldots, a_{n}\right\},
$$

where

$$
\left.\begin{array}{l}
z=-\operatorname{tr} A_{1} A_{2}, \\
x_{j}=-\operatorname{tr} A_{2} \cdots A_{j} \\
y_{j}=-\operatorname{tr} A_{1} A_{j}
\end{array}\right\} j=3, \ldots, n-1 .
$$

Proposition 5.1. Traces of all elements in $G(\mathscr{S})$ are rational functions in $\Sigma_{0 n}$. The parameters in $\Sigma_{0 n}$ satisfy a polynomial identity

$$
F_{0 n}\left(\Sigma_{0 n}\right)=F_{0 n}\left(z, x_{3}, y_{3}, \ldots, x_{n-1}, y_{n-1}, a_{1}, a_{2}, \ldots, a_{n}\right)=0,
$$

where, in each $a_{k}, k=2, \ldots, n, x_{n-1}$ and $y_{n-1}, F_{0 n}\left(\Sigma_{0 n}\right)$ is monic and has degree $2^{n-3}$

Proof. For $n=4$ the theorem is known and classical (see Section 3.1.) Our proof proceeds by induction on $n$. We assume that the proposition holds for $n$. Let $\mathscr{S}=$ $\left(A_{1}, \ldots, A_{n+1}\right)$ represent a point of $\mathscr{T}(0, n+1)\left(a_{1}, \ldots, a_{n+1}\right)$. If $B_{n}=A_{n} A_{n+1}$ and $b=-\operatorname{tr} B_{n}$, then $\mathscr{S}_{0}=\left\{A_{1}, \ldots, A_{n-1}, B_{n}\right\}$ is of type $(0, n)$ and traces of all elements in $G\left(\mathscr{S}_{0}\right)$ are rational functions in

$$
\Sigma_{0 n}=\left\{z, x_{3}, y_{3}, \ldots, x_{n-1}, y_{n-1}, a_{1}, \ldots, a_{n-1}, b\right\} .
$$

We also have an identity

$$
\begin{aligned}
& F_{0 n}\left(z, x_{3}, y_{3}, \ldots, x_{n-1}, y_{n-1}, a_{1}, \ldots, a_{n-1}, b\right) \\
& =b^{m}+\alpha_{m-1} b^{m-1}+\cdots+\alpha_{1} b+\alpha_{0}=0
\end{aligned}
$$


where $m=2^{n-3}$ and $\alpha_{j}$ are polynomials in $\Sigma_{0 n} \backslash\{b\}$. By our assumption $\alpha_{0}$ includes the sum $a_{2}^{m}+\cdots+a_{n-1}^{m}+x_{n-1}^{m}+y_{n-1}^{m}$. Let $C=A_{2} \cdots A_{n-1}$. Then the tuple $\left(A_{1}, C, A_{n}, A_{n+1}\right)$ is of type $(0,4)$ and its corresponding $\Sigma_{04}$ in (2.2) is

$$
\begin{array}{r}
\left\{-\operatorname{tr} C A_{n},-\operatorname{tr} A_{n} A_{1},-\operatorname{tr} A_{1} C,-\operatorname{tr} A_{1},-\operatorname{tr} C,-\operatorname{tr} A_{n},-\operatorname{tr} A_{n+1}\right\} \\
=\left\{x_{n}, y_{n}, b, a_{1}, x_{n-1}, a_{n}, a_{n+1}\right\} .
\end{array}
$$

We have (2.3) of the form

$$
F_{04}\left(x_{n}, y_{n}, b, a_{1}, x_{n-1}, a_{n}, a_{n+1}\right)=b^{2}+A b+B=0,
$$

where $A$ and $B$ are polynomials of $\left\{x_{n}, y_{n}, a_{1}, x_{n-1}, a_{n}, a_{n+1}\right\}$. From (2.3), for $x=a_{1}, x_{n-1}, a_{n}, a_{n+1}, x_{n}$ and $y_{n}, \operatorname{deg}_{x} A=1, \operatorname{deg}_{x} B=2$ and $B$ is monic, where $\operatorname{deg}_{x}$ means the degree in the variable $x$.

Dividing (5.2) by (5.3) results in the remainder $P b+Q=0$ with $P$ and $Q$ as in (5.6) below. From this and (5.3) we obtain a polynomial equation

$$
F_{0 n+1}\left(\Sigma_{0, n+1}\right)=B P^{2}-A P Q+Q^{2}=0 .
$$

In order to prove the last statement of Proposition 5.1 by induction, we must show

Lemma 5.1. $F_{0 n+1}\left(\Sigma_{0, n+1}\right)$ is a monic polynomial of degree $2 m=2^{n-2}$ in variables $a_{2}, \ldots, a_{n}, a_{n+1}, x_{n}$ and $y_{n}$.

Proof. From (5.2) and (5.3) we have

$$
\sum_{j=0}^{m} \alpha_{j} b^{j}=\left(\sum_{j=0}^{m-2} x_{j} b^{j}\right)\left(b^{2}+A b+B\right)+P b+Q
$$

with $\alpha_{m}=x_{m-2}=1$ and

$$
\left\{\begin{array}{l}
x_{m-3}+A x_{m-2}=\alpha_{m-1} \\
x_{j-2}+A x_{j-1}+B x_{j}=\alpha_{j}, \quad j=2, \ldots, m-2, \\
A x_{0}+B x_{1}+P=\alpha_{1} \\
B x_{0}+Q=\alpha_{0}
\end{array}\right.
$$

Let

$$
M=\left(\begin{array}{rr}
-A & 1 \\
-B & 0
\end{array}\right), L=\left(\begin{array}{rr}
1 & 0 \\
0 & -B
\end{array}\right)
$$

and ${ }^{t} M$ be the transpose of $M$. Then $L^{t} M=M L$ and

$$
M L\left(\begin{array}{l}
1 \\
0
\end{array}\right)=M^{2}\left(\begin{array}{l}
0 \\
1
\end{array}\right) \text {. }
$$

From (5.5) we have

$$
\left(\begin{array}{l}
P \\
Q
\end{array}\right)=M L\left(\begin{array}{l}
x_{0} \\
x_{1}
\end{array}\right)+\left(\begin{array}{l}
\alpha_{1} \\
\alpha_{0}
\end{array}\right),\left(\begin{array}{l}
x_{m-3} \\
x_{m-2}
\end{array}\right)={ }^{t} M\left(\begin{array}{l}
1 \\
0
\end{array}\right)+\left(\begin{array}{c}
\alpha_{m-1} \\
0
\end{array}\right)
$$

and

Therefore,

$$
\left(\begin{array}{c}
x_{j-2} \\
x_{j-1}
\end{array}\right)={ }^{t} M\left(\begin{array}{c}
x_{j-1} \\
x_{j}
\end{array}\right)+\left(\begin{array}{c}
\alpha_{j} \\
0
\end{array}\right), \quad j=2, \ldots, m-2 .
$$

$$
\left(\begin{array}{l}
P \\
Q
\end{array}\right)=\sum_{j=0}^{m} \alpha_{j}\left(\begin{array}{ll}
-A & 1 \\
-B & 0
\end{array}\right)^{j}\left(\begin{array}{l}
0 \\
1
\end{array}\right)
$$

with $\alpha_{m}=1$. Let $x$ stand for one of $a_{n}, a_{n+1}, x_{n}$ and $y_{n}$ and write $A=\alpha x+\beta$ and $B=x^{2}+\gamma x+\delta$ with $\alpha, \beta, \gamma$ and $\delta$ independent of $x$. By induction on $j$ we 
see the first and second entry of $M^{j}\left(\begin{array}{l}0 \\ 1\end{array}\right)$ for $j \geq 1$ are polynomials in $x$ of degree $j-1$ and $j$ with leading coefficients

$$
\left(\begin{array}{rr}
-\alpha & 1 \\
-1 & 0
\end{array}\right)^{j}\left(\begin{array}{l}
0 \\
1
\end{array}\right)
$$

respectively. Hence $\operatorname{deg}_{x} P=m-1$ and $\operatorname{deg}_{x} Q=m$. From (5.4) we see that

$$
\operatorname{deg}_{x} F_{0 n+1}=2 m=2^{n-2} .
$$

From (5.4) the leading coefficient of $\operatorname{deg}_{x} F_{0 n+1}$ in the variable $x$ is

$$
(0,1)\left(\begin{array}{cc}
-\alpha & -1 \\
1 & 0
\end{array}\right)^{m}\left(\begin{array}{cc}
1 & -\alpha / 2 \\
-\alpha / 2 & 1
\end{array}\right)\left(\begin{array}{cc}
-\alpha & 1 \\
-1 & 0
\end{array}\right)^{m}\left(\begin{array}{l}
0 \\
1
\end{array}\right)=1 .
$$

Here we used the fact that

$$
\left(\begin{array}{cc}
-\alpha & -1 \\
1 & 0
\end{array}\right)\left(\begin{array}{cc}
1 & -\alpha / 2 \\
-\alpha / 2 & 1
\end{array}\right)\left(\begin{array}{cc}
-\alpha & 1 \\
-1 & 0
\end{array}\right)=\left(\begin{array}{cc}
1 & -\alpha / 2 \\
-\alpha / 2 & 1
\end{array}\right) .
$$

Now we conclude $F_{0 n+1}=F_{0 n+1}\left(\Sigma_{0, n+1}\right)$ is monic polynomial of degree $2^{n-2}$ in variables $a_{n}, a_{n+1}$ and also in $x_{n}$ and $y_{n}$. By the induction hypothesis $F_{0 n}$ is monic and has degree $2^{n-3}$ in $a_{2}, \ldots, a_{n-1}$. The term $\alpha_{0}^{2}$ in $Q^{2}$ shows that $F_{0 n+1}$ is monic and has degree $2^{n-2}$ in $a_{2}, \ldots, a_{n-1}$.

We introduce auxiliary traces $z_{j}=-\operatorname{tr} A_{1} A_{2} \cdots A_{j-1}\left(\right.$ so $\left.b=z_{n}\right)$. Then, from a series of tuples,

$$
\left(A_{1},\left(A_{2} \cdots A_{j-2}\right), A_{j-1},\left(A_{j} \cdots A_{n+1}\right)\right), j=4, \ldots n+1,
$$

of type $(0,4)$, we obtain the equations

$$
F_{04}\left(x_{j-1}, y_{j-1}, z_{j-1}, a_{1}, x_{j-2}, a_{j-1}, z_{j}\right)=0, j=4, \ldots, n
$$

with $x_{2}=a_{2}, z_{3}=z$ and

$$
F_{04}\left(x_{n}, y_{n}, z_{n}, a_{1}, x_{n-1}, a_{n}, a_{n+1}\right)=0 .
$$

Starting with $\mathscr{R}=\mathscr{R}\left(\Sigma_{0, n+1} \backslash\left\{x_{n}\right\}\right)$, we have a sequence of quadratic extensions $\mathscr{R} \subset \mathscr{R}\left(z_{4}\right) \subset \mathscr{R}\left(z_{4}, z_{5}\right) \subset \cdots \subset \mathscr{R}\left(z_{4}, \ldots, z_{n}\right) \subset \mathscr{R}\left(z_{4}, \ldots, z_{n}, x_{n}\right)$, and hence, $x_{n}$ is in an extension of degree $2^{n-2}$ of the rational function field $\mathscr{R}\left(\Sigma_{0, n+1} \backslash\left\{x_{n}\right\}\right)$. Thus $F_{0 n+1}\left(\Sigma_{0, n+1}\right)$ in (5.4) is irreducible in $x_{n}$. Then the first equation in (5.4) shows that, as polynomials in $x_{n}, P$ and $Q$ are relatively prime. Therefore $P=Q=0$ occurs on a proper subvariety in the $2 n-4$ dimensional $\left(z, x_{3}, \ldots, x_{n-1}, y_{3}, \ldots, y_{n}\right)$ space. Since $b$ is continuous,

$$
b=-\operatorname{tr} A_{n} A_{n+1}=-Q / P
$$

throughout the Teichmüller space. Equation (5.7) shows that $b$ belongs to $\mathscr{R}\left(\Sigma_{0 n+1}\right)$ and hence $\mathscr{R}\left(\Sigma_{0 n}\right) \subset \mathscr{R}\left(\Sigma_{0 n+1}\right)$ (see (5.1) ).

In order to conclude the proof, by Lemma 2.3 it suffices to show that

$$
\operatorname{tr} A_{i} A_{j}, \quad(1 \leq i<j \leq n), \quad \operatorname{tr} A_{i} A_{j} A_{k}(1 \leq i<j<k \leq n)
$$

are rational functions in $\Sigma_{0 n+1}=\left\{z, x_{3}, \ldots, x_{n}, y_{3}, \ldots, y_{n}, a_{1}, \ldots, a_{n+1}\right\}$. This is true for $j<n$ or $k<n$ because $\mathscr{R}\left(\Sigma_{0 n}\right) \subset \mathscr{R}\left(\Sigma_{0 n+1}\right)$. We consider only $\operatorname{tr} A_{i} A_{j} A_{n}$, 
because $\operatorname{tr} A_{i} A_{n}$ can be treated in a similar way. Let $X=A_{i} A_{j}$ with $i<j<n$. By a repeated use of Lemma 3.2 , the tuple $\mathscr{S}_{0}=\left(A_{1}, X, Y, A_{n}, A_{n+1}\right)$ with

$$
\begin{aligned}
Y=X^{-1} A_{2} \cdots A_{n-1}=\left(A_{i} A_{j}\right)^{-1} A_{2}\left(A_{i} A_{j}\right) & \cdots\left(A_{i} A_{j}\right)^{-1} A_{i-1}\left(A_{i} A_{j}\right) \\
& \times\left(A_{j}^{-1} A_{i+1} A_{j}\right) \cdots\left(A_{j}^{-1} A_{j-1} A_{j}\right) A_{j+1} \cdots A_{n-1}
\end{aligned}
$$

is of type $(0,5)$. Its corresponding $\Sigma_{05}$ in (4.1) is

$$
\left\{x_{n-1}, u, v, x_{n}, y_{n}, a_{1}, x, y, a_{n}, a_{n+1}\right\},
$$

where $u=-\operatorname{tr} A_{1} Y, v=-\operatorname{tr} A_{1} X$. Since all of its elements are rational functions in $\Sigma_{0 n+1}$, so is $\operatorname{tr} X A_{n}=\operatorname{tr} A_{i} A_{j} A_{n}$ by Proposition 4.1.

5.2. Case of type $(1, n)$. Let $\mathscr{S}=\left(A, B, A_{1}, \ldots, A_{n}\right)$ represent a point of the Teichmüller space $\mathscr{T}(1, n)\left(a_{1}, \ldots, a_{n}\right)$ with $n \geq 2$. The matrices $A, B, A_{1}, \ldots, A_{n}$ in $S L(2, \mathbb{R})$ satisfy $A B A^{-1} B^{-1} A_{1} A_{2} \cdots A_{n}=1$ and $a_{j}=-\operatorname{tr} A_{j}$ for $j=1, \ldots, n$. The group $G(\mathscr{S})$ is generated by $A, B, A_{1}, A_{2}, \ldots, A_{n-1}$. We define

$$
\Sigma_{1 n}=\left\{a, b, z, x_{1}, y_{1}, \ldots, x_{n-1}, y_{n-1}, a_{1}, \ldots, a_{n}\right\},
$$

where

$$
\begin{aligned}
& a=\operatorname{tr} A, \quad b=\operatorname{tr} B, \quad z=\operatorname{tr} A B, \\
& x_{j}=\operatorname{tr} B A^{-1} B^{-1} A_{1} \cdots A_{j}, \quad y_{j}=\operatorname{tr} A A_{j}, \quad j=1, \ldots, n-1 .
\end{aligned}
$$

Proposition 5.2. Traces of all elements in $G(\mathscr{S})$ are rational functions in $\Sigma_{1 n}$. The traces in $\Sigma_{1 n}$ satisfy a polynomial identity

$$
F_{1 n}\left(\Sigma_{1 n}\right)=F_{1 n}\left(a, b, z, x_{1}, y_{1}, \ldots, x_{n-1}, y_{n-1}, a_{1}, \ldots, a_{n}\right)=0,
$$

where $F_{1 n}\left(\Sigma_{1 n}\right)$, in $a_{k}, k=2, \ldots, n, x_{n-1}$ and $y_{n-1}$, is monic and has degree $2^{n-1}$.

Proof. Let $k=-\operatorname{tr} A B A^{-1} B^{-1}$. Since $a=\operatorname{tr} A$, we apply Proposition 5.1 to the tuple $\mathscr{S}_{0}=\left(-A,-B A^{-1} B^{-1}, A_{1}, \ldots, A_{n}\right)$ of type $(0, n+2)$. Then we see that traces of all elements in the group $G\left(\mathscr{S}_{0}\right)$ are rational functions in

$$
\left\{k, x_{1}, y_{1}, \ldots, x_{n-1}, y_{n-1}, a, a_{1}, \ldots, a_{n}\right\},
$$

and hence in $\Sigma_{1 n}$, because $k=a b z-a^{2}-b^{2}-z^{2}+2$. The traces in $\Sigma_{1 n}$ satisfy

$$
F_{0 n+2}\left(a b z-a^{2}-b^{2}-z^{2}+2, x_{1}, y_{1}, \ldots, x_{n-1}, y_{n-1}, a, a, a_{1}, \ldots, a_{n}\right)=0 .
$$

We rewrite this identity as

$$
F_{1 n}\left(\Sigma_{1 n}\right)=F_{1 n}\left(a, b, z, x_{1}, y_{1}, \ldots, x_{n-1}, y_{n-1}, a_{1}, \ldots, a_{n}\right)=0 .
$$

Then $F_{1 n}\left(\Sigma_{1 n}\right)$ is monic and has degree $2^{n-1}=2^{(n+2)-3}$ in $a_{2}, \ldots, a_{n}, x_{n-1}$ and $y_{n-1}$. If $X=A_{i}$ or $X=A_{i} A_{j}$, where $1 \leq i, j<n$ and $i<j$, then the traces $A X$, $A B A^{-1} X, A B A^{-1} B^{-1} X$ are rational functions in $\Sigma_{1 n}$. That $\operatorname{tr} B X$ and $\operatorname{tr} A B X$ are also in $\mathscr{R}\left(\Sigma_{1 n}\right)$ follows from Proposition 4.2 .

\section{CASE OF TYPE $(g, n)$}

We consider tuples

$$
\mathscr{S}=\left(A_{1}, B_{1}, \ldots, A_{g}, B_{g}, K_{g+1}, \ldots, K_{g+n}\right)
$$

of type $(g, n)$ with $g \geq 1$. Even for $j=1, \ldots, g$, we define $K_{j}=\left[A_{j}, B_{j}\right]=$ $A_{j} B_{j} A_{j}^{-1} B_{j}^{-1}$. Then the matrices satisfy $K_{1} K_{2} \cdots K_{g+n}=1$. For $j=2, \ldots, g$, let

$$
\mathscr{H}_{j}=\left(A_{j}, B_{j}, C_{j}, D_{j}\right)
$$


where

$$
\begin{aligned}
& C_{j}=K_{j+1} \cdots K_{g+n} A_{1} \quad(j \geq 2), \\
& D_{j}=B_{1} A_{1}^{-1} B_{1}^{-1} K_{2} \cdots K_{j-1} \quad(j \geq 3), \quad D_{2}=B_{1} A_{1}^{-1} B_{1}^{-1} .
\end{aligned}
$$

Then $\mathscr{H}_{j}$ is a tuple of type $(1,2)$. We consider the following $6 g+3 n-5$ traces

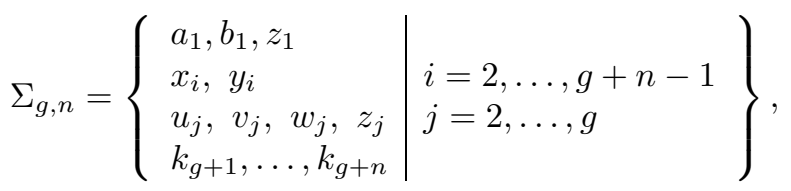

where

$$
\begin{aligned}
& a_{1}=\operatorname{tr} A_{1}, \quad b_{1}=\operatorname{tr} B_{1}, \quad z_{1}=\operatorname{tr} A_{1} B_{1}, \\
& x_{j}=\operatorname{tr} B_{1} A_{1}^{-1} B_{1}^{-1} K_{2} \cdots K_{j}, \quad y_{j}=\operatorname{tr} A_{1} K_{j}, \quad j=2, \ldots, g+n-1, \\
& \left.\begin{array}{ll}
u_{j}=\operatorname{tr} C_{j} A_{j} B_{j} A_{j}^{-1}, & v_{j}=\operatorname{tr} C_{j} A_{j} B_{j}^{2} \\
w_{j}=\operatorname{tr} C_{j} A_{j} B_{j}, & z_{j}=\operatorname{tr} A_{j} B_{j}
\end{array}\right\} j=2, \ldots, g,
\end{aligned}
$$

and $k_{j}=-\operatorname{tr} K_{j}$ for $j=1, \ldots, g+n$. We also let $x_{1}=\operatorname{tr} B_{1} A_{1}^{-1} B_{1}^{-1}=a_{1}$.

Proposition 6.1. Traces of all elements in $G(\mathscr{S})$ are rational functions in $\Sigma_{g, n}$. The parameters in $\Sigma_{g, n}$ satisfy a polynomial identity

$$
F_{g, n}\left(\Sigma_{g, n}\right)=0
$$

where $F_{g, n}\left(\Sigma_{g, n}\right)$ is monic and has degree $2^{2 g+n-3}$ in $k_{g+1}, \ldots, k_{g+n}, x_{g+n-1}$ and $y_{g+n-1}$.

Our proof of Proposition 6.1 is by induction on $j=1, \ldots, g$. The case for $j=1$ is treated in Section 5.2. At the $g$-th step we conclude the proof of the proposition. For $j \geq 1$, We define a tuple $\mathscr{S}_{j}$ of type $(j, g+n-j)$ :

$$
\mathscr{S}_{j}=\left(A_{1}, B_{1}, \ldots, A_{j-1}, B_{j-1}, A_{j}, B_{j}, K_{j+1}, \ldots, K_{g+n}\right)
$$

and

$$
\Sigma_{j, g+n-j}=\left\{\begin{array}{l|l}
a_{1}, b_{1}, z_{1} & \\
x_{i}, y_{i} & i=2, \ldots, g+n-1 \\
u_{k}, v_{k}, w_{k}, z_{k} & k=2, \ldots, j \\
k_{l} & l=j+1, \ldots, g+n
\end{array}\right\} .
$$

We assume that traces of all elements of the group generated by $\mathscr{S}_{j-1}$ are rational functions in $\Sigma_{j-1, g+n+1-j}$ and also that we have the polynomial identity

$$
F_{j-1, g+n+1-j}\left(\Sigma_{j-1, g+n+1-j}\right)=0,
$$

where the left-hand side is monic and has degree $2^{g+n+j-4}$ in $k_{j}, \ldots, k_{g+n}, x_{g+n-1}$ and $y_{g+n-1}$. By Proposition 4.3 traces of all elements in the group generated by $\mathscr{H}_{j}$, in particular, $a_{j}=\operatorname{tr} A_{j}$ and $b_{j}=\operatorname{tr} B_{j}$ are rational functions in $u_{j}, v_{j}, w_{j}, z_{j}$, $k_{j}, x_{j}$ and $x_{j-1}$. These seven parameters satisfy (4.12), which can be described as

$$
F_{12}\left(u_{j}, v_{j}, w_{j}, z_{j}, k_{j}, x_{j}, x_{j-1}\right)=k_{j}^{2}+A k_{j}+B=0,
$$

where $A$ and $B$ are polynomials in $\left\{u_{j}, v_{j}, w_{j}, z_{j}, x_{j}, x_{j-1}\right\}$. Now we proceed just as in the proof of Proposition 5.1. By dividing (6.4) by this polynomial (6.5) we obtain $P k_{j}+Q=0$, where $P$ and $Q$ are polynomials in

$$
\Sigma_{j, g+n-j}=\left(\Sigma_{j-1, g+n+1-j} \backslash\left\{k_{j}\right\}\right) \cup\left\{u_{j}, v_{j}, w_{j}, z_{j}\right\}
$$


and $Q$ is monic and has degree $2^{g+n+j-4}$ in $k_{j+1}, \ldots, k_{g+n}, x_{g+n-1}$ and $y_{g+n-1}$. We obtain also the polynomial identity

$$
F_{j, g+n-j}\left(\Sigma_{j, g+n-j}\right)=B P^{2}-A P Q+Q^{2}=0 .
$$

The left-hand side is monic and has degree degree $2^{g+n+j-3}$ in $k_{j+1}, \ldots, k_{g+n}$, $x_{g+n-1}$ and $y_{g+n-1}$. Let

$$
\mathscr{R}=\mathscr{R}\left(a_{1}, b_{1}, z_{1}, x_{2}, \ldots, x_{g+n-1}, y_{2}, \ldots, y_{g+n-2}, k_{2}, \ldots, k_{g+n}\right) .
$$

(Note that $y_{g+n-1}$ drops out of the variables.) We introduce

$$
\tau_{j}=-\operatorname{tr} K_{j} K_{j+1} \cdots K_{g+n}=-\operatorname{tr} K_{1} \cdots K_{j-1}, j=3, \ldots, g+n-1 .
$$

We have a series of $g+n-2$ tuples

$$
\begin{aligned}
& \left(A_{1},\left(B_{1} A_{1}^{-1} B_{1}^{-1}\right), K_{2},\left(K_{3} \cdots K_{g+n}\right)\right), \\
& \left(A_{1},\left(B_{1} A_{1}^{-1} B_{1}^{-1} K_{2} \cdots K_{j-1}\right), K_{j},\left(K_{j+1} \cdots K_{g+n}\right)\right), \quad j=3, \ldots, g+n-2, \\
& \left(A_{1},\left(B_{1} A_{1}^{-1} B_{1}^{-1} K_{2} \cdots K_{g+n-2}\right), K_{g+n-1}, K_{g+n}\right)
\end{aligned}
$$

of type $(0,4)$ which yield

$$
F_{04}\left(x_{2}, y_{2}, k_{1}, a_{1}, a_{1}, k_{2}, \tau_{3}\right)=0
$$

with $k_{1}=-\operatorname{tr} K_{1}=a_{1} b_{1} z_{1}-a_{1}^{2}-b_{1}^{2}-z_{1}^{2}+2$,

$$
F_{04}\left(x_{j}, y_{j}, \tau_{j}, a_{1}, x_{j-1}, k_{j}, \tau_{j+1}\right)=0, j=3, \ldots, g+n-2,
$$

and

$$
F_{04}\left(x_{g+n-1}, y_{g+n-1}, \tau_{g+n-1}, a_{1}, x_{g+n-2}, k_{g+n-1}, k_{g+n}\right)=0 .
$$

Then, starting with $\mathscr{R}$ in (6.7), we have a sequence of quadratic extensions $\mathscr{R} \subset$ $\mathscr{R}\left(\tau_{3}\right) \subset \mathscr{R}\left(\tau_{3}, \tau_{4}\right) \subset \cdots \subset \mathscr{R}\left(\tau_{3}, \ldots, \tau_{g+n-1}\right) \subset \mathscr{R}\left(\tau_{3}, \ldots, \tau_{g+n-1}, y_{g+n-1}\right)$, and hence, $y_{g+n-1}$ is in an extension of degree $2^{g+n-2}$ of the rational function field $\mathscr{R}$. By $j-1$ quadratic equations as in (6.5) with $j$ replaced by $2, \ldots, j$, we see that $y_{g+n-1}$ is in an extension of degree $2^{g+n+j-3}$ of $\mathscr{R}\left(\Sigma_{j, g+n-j}\right)$. Hence the polynomial in (6.6) is irreducible in $y_{g+n-1}$ and $P=Q=0$ occurs on a proper subvariety of $6 j+2(g+n-j)-6$ dimensional $\Sigma_{j, g+n-j} \backslash\left\{y_{g+n-1}\right\}$-space. By continuity of $k_{j}$, $k_{j}=-Q / P$ holds throughout the Teichmüller space. Now we conclude that $k_{j}$ is a rational function in $\Sigma_{j, g+n-j}$.

The traces of the elements in $T\left(\mathscr{S}_{j}\right)$ which are not yet known to be rational functions in $\Sigma_{j, g+n-j}$ are included in

$$
\operatorname{tr} X A_{j}, \quad \operatorname{tr} X B_{j}, \quad \operatorname{tr} X A_{j} B_{j}
$$

where $X$ runs over
(i) $A_{i}$
$1 \leq i<j$
(ii) $B_{i}, A_{i} B_{i}$,
$1 \leq i<j$
(iii) $A_{i_{1}} A_{i_{2}}$,
$1 \leq i_{1}<i_{2}<j$,
(iv) $A_{i_{1}} B_{i_{2}}, B_{i_{1}} A_{i_{2}}, B_{i_{1}} B_{i_{2}}$
$1 \leq i_{1}<i_{2}<j$,
(v) $K_{i}$,
$j<i \leq g+n$,
(vi) $K_{i_{1}} K_{i_{2}}$,
$j<i_{1}<i_{2} \leq g+n$,
(vii) $K_{i_{2}} A_{i_{1}}$,
$1 \leq i_{1}<j<i_{2} \leq g+n$,
(viii) $K_{i_{2}} B_{i_{1}}$,
$1 \leq i_{1}<j<i_{2} \leq g+n$. 
( $X$ in $\operatorname{tr} X A_{j} B_{j}$ may run over (i), $B_{i}$ in (ii) and (v).) Let $\mathscr{R}_{j}=\mathscr{R}\left(\Sigma_{j, g+n-j}\right)$. Our goal is to show that traces in (6.8) are in $\mathscr{R}_{j}$. Let $J_{1}=g+n-j+1$ and $J_{2}=g+n+j-2$. We define $\left(M_{1}, M_{2}, \ldots, M_{g+n+j}\right)$ by

$$
\left(M_{1}, \ldots, M_{J_{1}}\right)=\left(K_{j+1}, \ldots, K_{g+n}, A_{1}\right)
$$

and

$$
\left(M_{J_{1}+1}, \ldots, M_{g+n+j}\right)=\left(B_{1} A_{1}^{-1} B_{1}^{-1}, A_{2}, B_{2} A_{2}^{-1} B_{2}^{-1}, \ldots, A_{j}, B_{j} A_{j}^{-1} B_{j}^{-1}\right) .
$$

Then

$$
M_{1} M_{2} \cdots M_{J_{1}}=C_{j}, \quad M_{J_{1}+1} \cdots M_{J_{2}}=D_{j} .
$$

The tuple $\mathscr{S}^{\prime}=\left(M_{1}, \ldots, M_{g+n+j}\right)$ is of type $(0, g+n+j)$, because $G\left(\mathscr{S}^{\prime}\right)$ is the fundamental group of the surface obtained by cutting $S_{j, g+n-j}$ along $j$ loops determined by $A_{1}, \ldots, A_{j}$.

First we see that, if $U$ runs over

$$
M_{i}, \quad M_{i_{1}} M_{i_{2}}, \quad\left(1 \leq i, i_{1}, i_{2} \leq J_{2}, i_{1}<i_{2}\right)
$$

and if

$$
t_{1}=\operatorname{tr} U A_{j}, \quad t_{2}=\operatorname{tr} U B_{j} A_{j}^{-1} B_{j}^{-1}
$$

belong to $\mathscr{R}_{j}$, then so do the matrices in (6.8). The tuple $\left(A_{j}, B_{j}, U, V\right)$, where $V=U^{-1}\left[B_{j}, A_{j}\right]$, is of type $(1,2)$ by a repeated use of Lemma 3.2 . Since the trace of $U A_{j} B_{j} A_{j}^{-1} B_{j}^{-1}=U K_{j} \in G\left(\mathscr{S}_{j-1}\right)$ belongs to $\mathscr{R}_{j}$, Proposition 4.2 claims (\#) all traces of the elements in the group generated by $\left(A_{j}, B_{j}, U\right)$; in particular, $\operatorname{tr} U B_{j}$ and $\operatorname{tr} U A_{j} B_{j}$ are rational functions in $\Sigma_{j-1, g+n-j+1} \cup\left\{t_{1}, t_{2}\right\}$. Therefore, if $t_{1}$ and $t_{2}$ belong to $\mathscr{R}_{j}$, then so do $\operatorname{tr} U B_{j}$ and $\operatorname{tr} U A_{j} B_{j}$. Matrices $X$ in (i), (iii), (v), (vi) and (vii) are included in (6.9) and hence $\operatorname{tr} X B_{j}$ and $\operatorname{tr} X A_{j} B_{j}$ belong to $\mathscr{R}_{j}$.

The argument that we will repeat is as above: knowing that all traces of

$$
U, A_{i} B_{i} A_{i}^{-1} B_{i}^{-1} U=K_{i} U, A_{i} U=M_{J_{1}+2 i-2} U, B_{i} A_{i}^{-1} B_{i}^{-1} U=M_{J_{1}+2 i-1} U
$$

belong to $\mathscr{R}_{j}$, we apply Proposition 4.2 to the triple $\left(A_{i}, B_{i}, U\right)$ to conclude that all traces of the elements in the group generated by $\left(A_{i}, B_{i}, U\right)$, in particular, $\operatorname{tr} B_{i} U$ and $\operatorname{tr} A_{i} B_{i} U$ belong to $\mathscr{R}_{j}$. Let $Z \in\left\{A_{j}, B_{j}, A_{j} B_{j}\right\}$. For matrices in (ii), the claim (\#) enables us to apply this argument to $\left(A_{i}, B_{i}, Z\right)$. The conclusion is that $\operatorname{tr} B_{i} Z$ and $\operatorname{tr} A_{i} B_{i} Z \in \mathscr{R}_{j}$. The argument applied to $\left(A_{j}, B_{j}, W_{1} W_{2}\right)$ for $W_{1} \in\left\{A_{i_{1}}, B_{i_{1}} A_{i_{1}}^{-1} B_{i_{1}}^{-1}, K_{i_{1}}\right\}$ and $W_{2} \in\left\{A_{i_{2}}, B_{i_{2}} A_{i_{2}}^{-1} B_{i_{2}}^{-1}, K_{i_{2}}\right\}$ concludes that $\operatorname{tr} W_{1} W_{2} Z \in \mathscr{R}_{j}$. Then, for matrices in (iv) the argument applied to the triples $\left(A_{i_{2}}, B_{i_{2}}, Z A_{i_{1}}\right)$ and $\left(A_{i_{1}}, B_{i_{1}}, A_{i_{2}} Z\right)$ concludes $\operatorname{tr} A_{i_{1}} B_{i_{2}} Z$ and $\operatorname{tr} B_{i_{1}} A_{i_{2}} Z \in$ $\mathscr{R}_{j}$, respectively. Next apply the argument to $\left(A_{i_{2}}, B_{i_{2}}, Z W\right)$, where $W$ is $A_{i_{1}}$, $B_{i_{1}} A_{i_{1}}^{-1} B_{i_{1}}^{-1}$ and $K_{i_{1}}$. The conclusion is that $\operatorname{tr} W B_{i_{2}} Z \in \mathscr{R}_{j}$. This conclusion and the above argument applied to the triple $\left(A_{i_{1}}, B_{i_{1}}, B_{i_{2}} Z\right)$ conclude that $\operatorname{tr} B_{i_{1}} B_{i_{2}} Z \in$ $\mathscr{R}_{j}$. Finally for matrices in (viii) we can conclude likewise that $\operatorname{tr} K_{i_{2}} B_{i_{1}} Z \in \mathscr{R}_{j}$ from the triple $\left(A_{i_{1}}, B_{i_{1}}, Z K_{i_{2}}\right)$.

Now we show that $t_{1}$ and $t_{2}$ in (6.10) belong to $\mathscr{R}_{j}$. In what follows we write $(A, B, C, D)$ instead of $\left(A_{j}, B_{j}, C_{j}, D_{j}\right)$. We will repeat the same argument in the last part of the proof of Proposition 5.1. By a repeated use of Lemma 3.2 we see the following tuples are of type $(0,5)$ :

(1) For $X=M_{i}$ or $X=M_{i_{1}} M_{i_{2}}$, where $J_{1}<i, i_{1}, i_{2} \leq J_{2}, i_{1}<i_{2}$,

$$
\mathscr{S}_{1}^{*}=\left(C, D X^{-1}, X, A, B A^{-1} B^{-1}\right) \text {. }
$$


(2) For $X=M_{i}$ or $X=M_{i_{1}} M_{i_{2}}$, where $1 \leq i, i_{1}, i_{2} \leq J_{1}, i_{1}<i_{2}$,

$$
\mathscr{S}_{2}^{*}=\left(D, D^{-1} X D, D^{-1} X^{-1} C D, A, B A^{-1} B^{-1}\right) \text {. }
$$

(3) For $X=M_{i_{1}} M_{i_{2}}$, where $1 \leq i_{1} \leq J_{1}<i_{2} \leq J_{2}$,

$$
\mathscr{S}_{3}^{*}=\left(C M_{i_{1}}^{-1}, M_{i_{1}} D M_{i_{2}}^{-1} M_{i_{1}}^{-1}, X, A, B A^{-1} B^{-1}\right) .
$$

Case (1). We consider $\Sigma_{05}$ for $\mathscr{S}_{1}^{*}$ up to sign, which is

$$
\left\{\operatorname{tr} D, \operatorname{tr} C X, \operatorname{tr} C D X^{-1}, \operatorname{tr} A D, \operatorname{tr} A C, \operatorname{tr} C, \operatorname{tr} D X^{-1}, \operatorname{tr} X, \operatorname{tr} A, \operatorname{tr} A\right\} .
$$

Since $C, D, X \in G\left(\mathscr{S}_{j-1}\right)$ and $A, A C, A D \in G\left(\mathscr{H}_{j}\right)$, all traces in $\Sigma_{05}$ belong to $\mathscr{R}_{j}$. Then, by Proposition 4.1] $\operatorname{tr} X A$ and $\operatorname{tr} X B A^{-1} B^{-1}$ belong to $\mathscr{R}_{j}$.

Case (2). The tuple $\Sigma_{05}$ for $\mathscr{S}_{2}^{*}$ is

$$
\left\{\operatorname{tr} C, \operatorname{tr} X^{-1} C D, \operatorname{tr} X D, \operatorname{tr} D^{-1} C D A, \operatorname{tr} A D, \operatorname{tr} D, \operatorname{tr} X, \operatorname{tr} C X^{-1}, \operatorname{tr} A, \operatorname{tr} A\right\},
$$

up to sign. In this case again all traces in $\Sigma_{05}$ are in $\mathscr{R}_{j}$. As

$$
\begin{array}{ll}
X A=D\left(D^{-1} X D\right) D^{-1} A, & X B A^{-1} B^{-1}=D\left(D^{-1} X D\right) D^{-1} B A^{-1} B^{-1}, \\
X D A=D\left(D^{-1} X D\right) A, & C X^{-1} A=D\left(D^{-1} C X^{-1} D\right) D^{-1} A
\end{array}
$$

are elements of $G\left(\mathscr{S}_{2}^{*}\right)$, by Proposition 4.1 their traces belong to $\mathscr{R}_{j}$.

Case (3). Let $M=M_{i_{1}}$ and $N=M_{i_{2}}$. Then $X=M N$ and $\Sigma_{05}$ for $\mathscr{S}_{3}^{*}$ is

$$
\begin{aligned}
& \left\{\operatorname{tr} M D, \operatorname{tr} C N, \operatorname{tr} C D N^{-1} M^{-1}, \operatorname{tr} M D A, \operatorname{tr} C M^{-1} A,\right. \\
& \left.\quad \operatorname{tr} C M^{-1}, \operatorname{tr} D N^{-1}, \operatorname{tr} M N, \operatorname{tr} A, \operatorname{tr} A\right\}
\end{aligned}
$$

up to sign. Here $M D, C N, C D N^{-1} M^{-1}, C M^{-1}, N^{-1} D, M N$ are elements of $G\left(\mathscr{S}_{j-1}\right)$ and, as shown in Case $(2), \operatorname{tr} M D A, \operatorname{tr} C M^{-1} A$ belong to $\mathscr{R}_{j}$. Therefore, by Propostion 4.1, $\operatorname{tr} M N A$ and $\operatorname{tr} M N B A^{-1} B^{-1}$ belong to $\mathscr{R}_{j}$.

\section{ACTION OF THE MAPPING ClASS GROUP}

In this section we conclude the proof of Theorem 1.2 . Let $\mathcal{M}(g, n)$ be the mapping class group, the group of isotopy classes of orientation-preserving homeomorphisms of the surface of type $(g, n)$ which preserve every boundary component. Then $\mathcal{M}(g, n)$ acts on the Teichmüller space of type $(g, n)$ by a change of marking. Let $\mathcal{N}$ denote the normal subgroup of $\mathcal{M}(g, n)$ of mapping classes which fixes all points of the Teichmüller space. Then $\operatorname{Mod}(g, n)=\mathcal{M}(g, n) / \mathcal{N}$ is called the Teichmüller modular group. With a finite number of exceptional cases of $(g, n), \mathcal{M}(g, n)=$ $\operatorname{Mod}(g, n)($ see p. 203 of [22]).

Let $\operatorname{Out}(G(g, n))$ be the group of outer automorphisms of $G(g, n) \cdot \operatorname{Mod}(g, n)$ is embedded in Out $(G(g, n))$ as a subgroup. This subgroup is characterized by Nielsen (see [22, 11.3]). Hence, if $\varphi \in \operatorname{Mod}(g, n)$ and if $\mathscr{S}=\left(A_{1}, \ldots, A_{m}\right), m=2 g+n$, represents a general point of $\mathscr{T}=\mathscr{T}(g, n)\left(c_{1}, \ldots, c_{n}\right)$, then, up to simultaneous conjugations, the action of $\varphi$ on the Teichmüller space can be written in the form

$$
\varphi\left(A_{1}, \ldots, A_{m}\right)=\left(\varphi_{1}\left(A_{1}, \ldots, A_{m}\right), \ldots, \varphi_{N}\left(A_{1}, \ldots, A_{m}\right)\right),
$$

where $\varphi_{j}\left(A_{1}, \ldots, A_{m}\right) \in G(\mathscr{S})$. Then, by using the parameters $\chi=\left(x_{1}, \ldots, x_{N}\right)$, $N=6 g+2 n-5$, for $\mathscr{T}$ obtained so far, we can find rational functions

$$
f_{j}\left(x_{1}, \ldots, x_{N}\right)=\operatorname{tr} \varphi_{j}\left(A_{1}, \ldots, A_{m}\right), j=1, \ldots, N,
$$


with coefficients in $\mathbb{Q}\left(c_{1}, \ldots, c_{n}\right)$. Thus $\varphi$ induces a rational transformation

$$
F_{\varphi}\left(x_{1}, \ldots, x_{N}\right)=\left(f_{1}\left(x_{1}, \ldots, x_{N}\right), \ldots, f_{N}\left(x_{1}, \ldots, x_{N}\right)\right)
$$

on $\mathscr{T}(g, n)\left(c_{1}, \ldots, c_{n}\right)$ embedded in $\mathbb{R}^{N}$.

Remark. Let $Q(g, n)$ be the space of quasifuchsian structures on a Riemann surface of genus $g$ with $n$ punctures. Then $Q(g, n)$ is a fiber space over $\mathscr{T}(g, n)(2,2, \ldots, 2)$. The fiber over $X$ is identified with the Bers embedding of the Teichmüller space of the Riemann surface represented by $X$. Hence $Q(g, n)$ is simply connected and every trace function on $\mathscr{T}(g, n)(2,2, \ldots, 2)$ extends to $Q(g, n)$. Consequently, Theorem 1.2 can be extended to $Q(g, n)$.

\section{ACKNOWLEDGMENTS}

The authors thank the referee for many valuable comments on this paper.

\section{REFERENCES}

[1] A. F. Beardon, The geometry of discrete groups, Graduate Texts in Mathematics, vol. 91, Springer-Verlag, New York, 1983. MR698777

[2] M. Culler, Lifting representations to covering groups, Adv. in Math. 59 (1986), no. 1, 64-70, DOI 10.1016/0001-8708(86)90037-X. MR825087

[3] J. Gilman and B. Maskit, An algorithm for 2-generator Fuchsian groups, Michigan Math. J. 38 (1991), no. 1, 13-32, DOI 10.1307/mmj/1029004258. MR 1091506

[4] L. Keen, On Fricke moduli, Advances in the Theory of Riemann Surfaces (Proc. Conf., Stony Brook, N.Y., 1969), Ann. of Math. Studies, No. 66. Princeton Univ. Press, Princeton, N.J., 1971, pp. 205-224. MR0288252

[5] I. Kra, On lifting Kleinian groups to $\mathrm{SL}(2, \mathbf{C})$, Differential geometry and complex analysis, Springer, Berlin, 1985, pp. 181-193. MR.780044

[6] F. Luo, Geodesic length functions and Teichmüller spaces, J. Differential Geom. 48 (1998), no. 2, 275-317. MR1630186

[7] C. Maclachlan and A. W. Reid, The arithmetic of hyperbolic 3-manifolds, Graduate Texts in Mathematics, vol. 219, Springer-Verlag, New York, 2003. MR.1937957

[8] G. Nakamura and T. Nakanishi, Parametrizations of some Teichmüller spaces by trace functions, Conform. Geom. Dyn. 17 (2013), 47-57, DOI 10.1090/S1088-4173-2013-00254-3. MR.3037875

[9] T. Nakanishi and M. Näätänen, Parametrization of Teichmüller space by length parameters, Analysis and topology, World Sci. Publ., River Edge, NJ, 1998, pp. 541-560. MR1667832

[10] T. Nakanishi and M. Näätänen, Areas of two-dimensional moduli spaces, Proc. Amer. Math. Soc. 129 (2001), no. 11, 3241-3252, DOI 10.1090/S0002-9939-01-06010-5. MR.1844999

[11] Y. Okumura, On the global real analytic coordinates for Teichmüller spaces, J. Math. Soc. Japan 42 (1990), no. 1, 91-101, DOI 10.2969/jmsj/04210091. MR.1027542

[12] Y. Okumura, Global real analytic length parameters for Teichmüller spaces, Hiroshima Math. J. 26 (1996), no. 1, 165-179. MR.1380431

[13] R. C. Penner, The decorated Teichmüller space of punctured surfaces, Comm. Math. Phys. 113 (1987), no. 2, 299-339. MR919235

[14] N. Purzitsky, Two-generator discrete free products, Math. Z. 126 (1972), 209-223. MR.0346070

[15] P. Schmutz, Die Parametrisierung des Teichmüllerraumes durch geodätische Längenfunktionen (German), Comment. Math. Helv. 68 (1993), no. 2, 278-288, DOI 10.1007/BF02565819. MR1214232

[16] M. Seppälä and T. Sorvali, On geometric parametrization of Teichmüller spaces, Ann. Acad. Sci. Fenn. Ser. A I Math. 10 (1985), 515-526, DOI 10.5186/aasfm.1985.1058. MR802516

[17] M. Seppälä and T. Sorvali, Parametrization of Teichmüller spaces by geodesic length functions, Holomorphic functions and moduli, Vol. II (Berkeley, CA, 1986), Math. Sci. Res. Inst. Publ., vol. 11, Springer, New York, 1988, pp. 267-284, DOI 10.1007/978-1-4613-9611-6_18. MR.955845 
[18] M. Seppälä and T. Sorvali, Parametrization of Möbius groups acting in a disk, Comment. Math. Helv. 61 (1986), no. 1, 149-160, DOI 10.1007/BF02621907. MR847525

[19] M. Seppälä and T. Sorvali, Traces of commutators of Möbius transformations, Math. Scand. 68 (1991), no. 1, 53-58. MR1124819

[20] M. Seppälä and T. Sorvali, Geometry of Riemann surfaces and Teichmüller spaces, NorthHolland Mathematics Studies, vol. 169, North-Holland Publishing Co., Amsterdam, 1992. MR 1202043

[21] S. A. Wolpert, Geodesic length functions and the Nielsen problem, J. Differential Geom. 25 (1987), no. 2, 275-296. MR880186

[22] H. Zieschang, Finite groups of mapping classes of surfaces, Lecture Notes in Mathematics, vol. 875, Springer-Verlag, Berlin, 1981. MR643627

Science Division, Center for General Educhtion, Aichi Institute of Technology, 1247

YACHIGUSA, YAKUSA, TOYOTA, 470-0392, JAPAN

E-mail address: gou@aitech.ac.jp

Department of Mathematics, Shimane University, Matsue, 690-8504, Japan

E-mail address: tosihiro@riko.shimane-u.ac.jp 